RUNNING HEAD: WITHDRAWAL SYMPTOMICS

\title{
More than the sum of its parts: A network perspective on tobacco withdrawal
}

Lydon-Staley, D.M. ${ }^{*}$, Leventhal, A.M. ${ }^{2,3}$, Piper, M.E. ${ }^{4}$, Schnoll, R.A. ${ }^{5,6}$, \& Bassett, D.S. ${ }^{1,5,7,8,9,10}$

${ }^{1}$ Department of Bioengineering, School of Engineering and Applied Science, University of Pennsylvania

${ }^{2}$ Department of Preventive Medicine, Institute for Addiction Science, University of Southern California Keck School of Medicine

${ }^{3}$ Department of Psychology, University of Southern California

${ }^{4}$ Department of Medicine, University of Wisconsin Center for Tobacco Research and Intervention, University of Wisconsin School of Medicine

and Public Health

${ }^{5}$ Department of Psychiatry, Perelman School of Medicine, University of Pennsylvania

${ }^{6}$ Abramson Cancer Center, University of Pennsylvania

${ }^{7}$ Department of Electrical \& Systems Engineering, School of Engineering and Applied Science, University of Pennsylvania

${ }^{8}$ Department of Neurology, Perelman School of Medicine, University of Pennsylvania

${ }^{9}$ Department of Physics \& Astronomy, College of Arts and Sciences, University of Pennsylvania ${ }^{10}$ The Santa Fe Institute

*Corresponding author:

David M. Lydon-Staley, 210 S. 33 ${ }^{\text {rd }}$ Street, 240 Skirkanich Hall, Philadelphia, PA 19104-6321

Email: dlsta@seas.upenn.edu 
WITHDRAWAL SYMPTOMICS

\begin{abstract}
A recently developed network perspective on tobacco withdrawal posits that withdrawal symptoms causally influence one another across time, rather than simply being indicators of a latent syndrome. Evidence supporting a network perspective would shift the focus of tobacco withdrawal research and intervention toward studying and treating individual withdrawal symptoms and inter-symptom associations. Yet, key questions remain to be addressed, including whether withdrawal symptoms and their interactions are moderated by abstinence and by cessation treatment. Here we examine the evidence for a network perspective on tobacco withdrawal. First, we use data from 668 participants (40\% female, 78.29\% African American) reporting on their experiences of withdrawal after 16 hours of smoking abstinence and during a non-abstinent state to examine the extent to which individual withdrawal symptoms are differentially impacted by smoking deprivation. Second, we estimate the symptom network structure of tobacco withdrawal during states of smoking satiety and abstinence. Third, we use experience-sampling data from 1210 participants (58.35\% female, $86.24 \%$ white) undergoing smoking cessation treatment to estimate dynamic symptom networks that describe the interplay among withdrawal symptoms across time. Results indicate (i) differential impact of smoking deprivation on individual withdrawal symptoms, suggesting that symptoms are not interchangeable, (ii) substantial interplay among withdrawal symptoms that shows no evidence of change across periods of smoking satiety and abstinence, and (iii) reductions in the interplay among withdrawal symptoms during smoking cessation treatment. Overall, findings suggest the utility of a network perspective and encourage a greater consideration of the individual symptoms of tobacco withdrawal.
\end{abstract}

Keywords: networks; withdrawal; nicotine; tobacco; symptoms 
WITHDRAWAL SYMPTOMICS

\section{Introduction}

During efforts to quit smoking, aversive withdrawal symptoms appear that are prominent as primary motives for the resumption of smoking in many theories of addiction (Baker et al., 2004; Piper, 2015; Solomon \& Corbit, 1974). Withdrawal symptoms are often treated as interchangeable indicators of an underlying syndrome (Toll et al., 2007). During diagnosis (American Psychiatric Association, 2013), for example, criteria for tobacco withdrawal is met if a patient presents with four or more withdrawal symptoms (anger, anxiety, depressed mood, difficulty concentrating, increased appetite, insomnia, and restlessness). Yet, there is increasing evidence that individual withdrawal symptoms are not interchangeable; rather, they exhibit distinct time profiles during the course of cessation attempts (Hendricks et al., 2006; Leventhal et al., 2010; Piasecki et al., 2000; West, Hajek, \& Belcher, 1987) and unique responses to treatment (Foulds et al., 2013).

A network approach to tobacco withdrawal has recently been proposed to complement a syndrome perspective on withdrawal (Lydon-Staley et al., 2018). A network approach to psychopathology suggests that, rather than being passive indicators of a latent disorder, symptoms of a disorder interact to form networks of causally connected symptoms (Borsboom, 2017; Bringmann et al., 2013; Schmittman et al., 2013). From this perspective, the experience of tobacco withdrawal can be described as a network in which the nodes of the network represent symptoms and the edges (or lines between nodes) represent causal influences among symptoms across time. Studies provide preliminary support for the network perspective's proposal that individual tobacco withdrawal symptoms influence one another in a potentially causal fashion over time. For example, positive associations between anhedonia, negative affect, and craving have been observed over the course of a smoking cessation attempt (Cook et al., 2017) and 
WITHDRAWAL SYMPTOMICS

strong positive associations have been observed between sleep problems and restlessness, as well as among affective symptoms (anger, anxiety, and depressed mood), in smokers undergoing smoking cessation treatment (Lydon-Staley et al., 2018). Recent work in experience sampling has considered the dynamic associations among cessation fatigue, negative affect, nicotine craving, and self-efficacy in smokers undergoing cessation treatment (Bekiroglu et al., 2017). This work, which makes use of the temporal precedence available in time series data to model how the reporting of symptoms at a previous time point $(t-1)$ are associated with symptom intensity at the current time point $(t)$, found that these four constructs changed over time in response to each other during the course of smoking cessation.

While there is empirical support for the notion that individual symptoms of withdrawal influence one another over time, key questions about a network approach to withdrawal remain to be addressed, including whether withdrawal symptoms and their interactions are moderated by abstinence and by cessation treatment. The goal of this paper is to provide a thorough examination of the potential for a network perspective to inform understanding of tobacco withdrawal. First, the network perspective emphasizes the importance of considering individual symptoms in their own right. As such, we examine differences in the effects of acute smoking abstinence (16 hours) on individual withdrawal symptoms. We hypothesize that withdrawal symptom intensity will be increased during smoking abstinence relative to satiety, but that the magnitude of this increase will show marked variability across the many symptoms of withdrawal.

Second, we seek to provide insight into how individual withdrawal symptoms may influence one another across time and how levels of smoking satiety may impact these intersymptom associations. Accordingly, we employ network modeling techniques to estimate the 
WITHDRAWAL SYMPTOMICS

symptom network structure of tobacco withdrawal in a sample of tobacco smokers during smoking abstinence and during a non-abstinent session. Previous work modeling tobacco withdrawal symptom networks used 525 participants providing data at four time points over the course of 8 weeks of treatment with nicotine patch and behavioral counseling to evaluate the conditional dependence among seven symptoms (Lydon-Staley et al., 2018). Findings indicated minimal differences in the structure of withdrawal networks over the course of treatment. Yet, the treatment context within which data were collected may have impacted network stability. Here, we use an acute smoking abstinence laboratory paradigm to provide a stringent test for invariance in symptom network structure across smoking satiety and abstinence.

Third, we use experience-sampling data from participants providing momentary reports of withdrawal symptoms over 14 days to estimate a temporal network that describes the momentto-moment interplay among withdrawal symptoms. The use of experience-sampling data to construct temporal networks (Holme \& Saramäki, 2012) is an important complement to existing studies modeling cross-sectional withdrawal networks. The edges in cross-sectional networks indicate between-person associations among symptoms, with positive edges indicating that if, for example, an individual experiences high levels of anxiety, then they are also likely to experience high levels of anger. This symptom co-occurrence, from a network perspective, is theorized to result from causal associations among symptoms. Yet, between-person associations (i.e., people high in anxiety experience high levels of anger) do not always translate to the within-person associations (i.e., at times when a person is more anxious than usual, they are also angrier than usual; Bos et al., 2017; Hamaker, Dolan, \& Molenaar, 2005). One of the strengths of the network approach is that it may incorporate within-person as well as between-person associations. 
WITHDRAWAL SYMPTOMICS

Further, the use of temporal networks allows a test for the presence of a potential source of distress experienced during smoking cessation, unique to a network approach to withdrawal, that may undermine efforts to remain abstinent. In particular, individuals undergoing cessation may experience continued symptom activation long after the cause of symptom activity (i.e., deprivation) has disappeared due to symptom interplay across time (Borsboom, 2017; Cramer et al., 2016). This failure for symptoms to de-activate arises in strongly connected symptom networks that facilitate the reverberation of symptom activity through the network across time as activity in one symptom is transferred to another symptom via causal associations. Evidence for self-perpetuating symptom networks and their association with psychopathology comes from studies showing that individuals with densely connected symptom networks are more likely to experience greater symptoms of depression (Yang et al., 2018; Lydon-Staley et al., 2019a; Pe et al., 2015; Bello et al., 2017). For example, individuals with networks of socioemotional states (e.g., anger, shame, and perceived control) structured such that they facilitate the persistent experience of sadness when perturbed show higher levels of depressive symptoms (Yang et al., 2018). Such an explanation would fit with empirical evidence of extended withdrawal symptom experiences among some smokers (Piasecki et al., 1998; 2003) when other research suggests that withdrawal should peak after the first 14 days (Hughes, 2007). The generalizability of the phenomenon of self-perpetuating symptom networks to tobacco withdrawal awaits empirical examination. We estimate person-specific networks articulating the moment-to-moment associations between withdrawal symptoms across time and quantify individual differences in the extent to which individual networks facilitate the reverberation of symptom activity through participants' networks. We test whether participants undergoing smoking cessation treatment show less symptom reverberation across time relative to participants in a placebo condition. We 
WITHDRAWAL SYMPTOMICS

hypothesize that treatment hinders the reverberation of symptom activity across time and the experience of self-perpetuating symptom networks during smoking cessation resulting in weaker relations between symptoms.

\section{Study 1}

A network perspective on withdrawal suggests that individual symptoms are meaningful in their own right and that they causally influence one another over time. To test the extent to which individual withdrawal symptoms may be considered interchangeable, we examined changes in the intensity of individual withdrawal symptoms across smoking abstinent (16 hours of abstinence) and non-abstinent sessions in 668 daily smokers. We hypothesized that, while many withdrawal symptoms will demonstrate increases in intensity during smoking abstinence relative to non-abstinence, the extent of change in intensity will differ across individual withdrawal symptoms. We then examined the network structure of tobacco withdrawal symptoms and the extent to which it varied across periods of smoking abstinence and nonabstinence. Based on previous work that indicated limited changes in withdrawal network structure across a smoking cessation attempt (Lydon-Staley et al., 2018), we hypothesized that there would be minimal differences in network structure across the smoking non-abstinent and smoking abstinent sessions.

\section{Method}

\section{Participants}

Data were drawn from an ongoing study of individual differences in the expression of tobacco withdrawal in African Americans, which included a small comparison sample of European Americans (Bello et al., 2017). Participants ( $n=668 ; 40 \%$ female; $78.29 \%$ African 
WITHDRAWAL SYMPTOMICS

American) were non-treatment seeking daily cigarette smokers (Mean age $=48.99$ years,

$S D=11.18$ ) recruited from the metropolitan Los Angeles area via word of mouth and by both newspaper and online advertisements. Study inclusion criteria were: 18 years of age or older; daily cigarette smoking for at least the past 2 years; typically smokes $\geq 10$ cigarettes per day; self-report being African American (or European American), and fluent in English. Study exclusion criteria were: current $D S M-I V$ non-nicotine substance dependence; breath carbon monoxide $(\mathrm{CO})$ levels $<10 \mathrm{ppm}$ at intake; desire to substantially cut down or quit smoking in the next 30 days; current use of anti-depressant, psychostimulant, or anti-psychotic medications; use of anxiolytic medications more than once per week; reporting being pregnant or breast-feeding; and daily use of other non-cigarette tobacco products or nicotine replacement therapy. Participants were compensated \$200. All study procedures were approved by the University of Southern California Internal Review Board.

\section{Procedure}

An initial phone screening determined preliminary eligibility. Potentially eligible participants then attended an initial laboratory session involving additional in-person screening and assessments. The initial session included informed consent, analysis of breath $\mathrm{CO}$ and blood alcohol, completion of the Structured Clinical Interview for DSM-IV Research Edition (23) substance use disorder module to determine eligibility, and completion of additional assessments. Participants then attended two counterbalanced experimental sessions starting around noon: one abstinent and one non-abstinent ad libitum smoking.

Both experimental sessions followed identical procedures with the following exception: for the non-abstinent session, participants were instructed to smoke as they normally would prior to their visit and then were required to smoke a cigarette of their preferred brand in the 
WITHDRAWAL SYMPTOMICS

laboratory at session outset (to standardize for smoking recency). For the abstinent session, by

contrast, participants were instructed not to smoke after $8 \mathrm{pm}$ the night before their visit (16 hours of abstinence). Upon arrival at the laboratory (abstinent session) or following smoking their preferred brand of cigarette (non-abstinent session), breath $\mathrm{CO}(\leq 9 \mathrm{ppm}$ for abstinent ssession) and alcohol $(\mathrm{BrAC}=0.00$ required for session continuation) assessments were conducted. Following $\mathrm{CO}$ and alcohol assessments, participants answered a set of questions designed to assess withdrawal symptoms.

\section{Measures}

We used the Minnesota and Nicotine Withdrawal Scale (MNWS; Hughes \& Hatsukami, 1986) to measure tobacco withdrawal. The scale instructs participants to rate their craving, irritability, anxiety, concentration problems, restlessness, impatience, hunger, cardiovascular and autonomic activation, drowsiness, and headaches experienced "so far today" on a 6-point scale ranging from "none" to "severe".

\section{Analysis}

To examine the extent to which individual withdrawal symptoms were differentially impacted by smoking satiety we used Wilcoxon tests for paired samples to examine differences in symptom intensity for all withdrawal symptoms across the abstinent and non-abstinent sessions. A Wilcoxon test was used to accommodate the ordinal nature of the data. We calculated effect sizes to quantify the magnitude of change in symptom intensity by dividing the standardized $z$-statistic of the Wilcoxon test by the square root of the number of participants. 
WITHDRAWAL SYMPTOMICS

To examine the network structure of tobacco withdrawal symptoms, we analyzed complete data available from the MNWS during the abstinent $(\mathrm{n}=665)$ and non-abstinent $(n=668)$ assessments. For each assessment, we estimated a tobacco withdrawal network using polychoric correlations of the ordinal withdrawal measures to create a covariance matrix which served as the input to a Gaussian graphical model (GGM; Epskamp \& Fried, 2018; Lauritzen, 1996). In this GGM, nodes represent individual symptoms of tobacco withdrawal. Nodes are connected by undirected edges indicating conditional dependence between two symptoms. The use of the GGM entails the estimation of many parameters. To avoid obtaining false positive associations among symptoms, we used a regularization approach - the graphical least absolute shrinkage and selection operator (LASSO) - to shrink all edge weights, setting many to zero (Epskamp et al., 2012). This approach mitigates the problem of estimating spurious associations and results in a sparse network structure containing only likely connections.

To investigate differences in the structure of the tobacco withdrawal network across the two assessment periods, we used a permutation-based hypothesis test named the Network Comparison Test (NCT; van Borkulo et al., 2015) using 1000 iterations. We tested for differences in network structure (i.e., network structure invariance) and in the overall level of connectivity (i.e., global strength invariance) between abstinent and non-abstinent measurement occasions.

Additional analyses investigating the accuracy of network estimation as well as measures of node centrality are available in the supplement (Study 1: Supplemental Analysis and Results). Nodes with high centrality have strong connections to many other nodes and connect otherwise disparate nodes to one another. As such, they are theorized to be particularly influential in the 
WITHDRAWAL SYMPTOMICS

development and maintenance of mental disorders (Borsboom \& Cramer, 2013; Fried et al., 2016; but see Bringmann et al., 2019; Hallquist, Wright, \& Molenaar, 2019).

\section{Results}

Participants reported a median Fagerstrom Test of Nicotine Dependence classification of high dependence and, at baseline, reported smoking an average of $15.18(S D=7.16)$ cigarettes per day on a normal day. An examination of breath $\mathrm{CO}$ levels across smoking non-abstinent

$(M=18.49, S D=10.11)$ and smoking abstinent $(M=4.84, S D=2.51)$ sessions to confirm the success of the experimental manipulation of smoking satiety revealed significantly decreased CO during the abstinent session relative to the non-abstinent session, $t(658)=-36.43, p<0.001$.

The Wilcoxon signed-rank tests revealed significant increases in the intensity of seven individual withdrawal symptoms during abstinent sessions compared to non-abstinence sessions: craving, anger/irritability, anxious/tense mood, difficulty concentrating, restlessness, excessive hunger, and headache (all $p$-values $<0.001$; Fig. 1). There were no significant changes in autonomic symptoms or drowsiness between the abstinent and non-abstinent sessions (all $p$-values $>0.05$ ). Increases in the experience of headache were small in magnitude $(r=0.22)$; increases in difficulty concentrating $(r=0.46)$, excessive hunger $(r=0.45)$, and restlessness $(r=0.45)$ were medium in magnitude; and increases in anxious mood $(r=0.58)$, anger $(r=0.58)$, and craving $(r=0.65)$ were large in magnitude. Results using a subsample $(n=523)$ of the dataset consisting of only AfricanAmerican participants can be found in the supplement (Study 1: Individual withdrawal symptoms are differentially impacted by smoking deprivation: African American subsample), and also indicate differential impacts of acute smoking abstinence on individual withdrawal symptoms in a similar manner to that observed in the combined sample. 
WITHDRAWAL SYMPTOMICS

[FIGURE 1 ABOUT HERE]

The estimated tobacco withdrawal symptom networks at the smoking abstinent and nonabstinent sessions are shown in Figure 2 (see supplemental Tables S1 and S2 for the associated adjacency matrices). At each assessment, symptoms of tobacco withdrawal were highly interconnected, with associations emerging among most symptoms. Of 36 potential edges, 6 were non-zero during both the non-abstinent session and the abstinent session. There were also 3 edges estimated to be zero across both abstinent and non-abstinent sessions: the edges between headaches and craving, difficulty concentrating and excessive hunger, and restlessness and headaches.

Most edges were positive (depicted in blue), indicating that participants experiencing high levels of a particular symptom were likely to show high levels of other symptoms connected to it. Four negative edges (depicted in red) were observed in the non-abstinent network, indicating that participants with greater craving experienced less drowsiness and fewer physical symptoms (tremors, heart racing, etc.). Additionally, participants experiencing greater anxiety or tension reported less excessive hunger and less drowsiness. The abstinent network contained two negative edges: participants with greater craving experienced less drowsiness and fewer physical symptoms (tremors, heart racing, etc.), consistent with the findings during the non-abstinent session.

[FIGURE 2 ABOUT HERE] 
WITHDRAWAL SYMPTOMICS

The pairwise Network Comparison Tests of network structure and global connectivity invariance indicated no significant differences in network structure or global strength across the abstinent and non-abstinent sessions (all $p$-values $>0.05$; Fig. S1). This result suggests that the structure of tobacco withdrawal networks and the strength of the relations among symptoms are relatively stable during non-abstinent and abstinent states. Results using a subsample $(n=523)$ of the full dataset consisting of African-American participants can be found in the supplement and also indicate no significant differences in network structure or global strength across the abstinent and non-abstinent sessions (Study 1: The network structure of tobacco withdrawal across deprived and nondeprived sessions: African-American subsample). The results of additional analyses examining the accuracy of network estimation as well as the centrality of individual symptoms can be found in the supplement (Study 1: Supplemental Results).

\section{Study 2}

To examine dynamic networks that articulate the moment-to-moment interactions among withdrawal symptoms over time, we analyzed time series of tobacco withdrawal symptoms obtained from 1210 participants who reported symptom intensity four times a day for 14 days during a smoking cessation attempt.

\section{Method}

\section{Participants}

Participants were 1210 smokers (58.35\% female, 86.24\% white), previously reported on in an existing study (Piper et al., 2009; Bolt et al., 2012). Participants were recruited via TV, radio, and newspaper advertisements, community flyers, and earned media (e.g., radio and TV 
WITHDRAWAL SYMPTOMICS

interviews, press releases) in the greater Madison and Milwaukee (Wisconsin) areas. The primary inclusion criteria were smoking at least 10 cigarettes per day for the past 6 months and being motivated to quit smoking. Exclusion criteria included: use of certain medications (including MAO inhibitors, bupropion); any history of psychosis, bipolar disorder, or an eating disorder; consuming six or more alcohol beverages daily 6 or 7 days a week; pregnancy or breast-feeding; and a serious health condition that might prevent study completion.

\section{Procedure}

Participants passing a phone screen were invited to an information session at which a study description was provided and written informed consent was obtained. Participants then completed multiple baseline screenings, including a medical history screening, vital signs measurements, and a CO breath test. Participants also completed demographic, smoking history, and tobacco dependence questionnaires.

Eligible participants were randomized to one of six treatment conditions: bupropion sustained-release (SR) $(n=215)$, nicotine lozenge $(n=202)$, nicotine patch $(n=210)$, nicotine patch + nitocine lozenge $(n=228)$, buproprion SR + nicotine lozenge $(n=214)$, or placebo $(n=141)$. Five placebo conditions matched the five active conditions, with three monotherapy placebo conditions $(n=80)$ and two combination therapy placebo conditions $(n=61)$. All medications were provided for 8 weeks post-quit except the nicotine lozenge, which was provided for 12 weeks post-quit, consistent with prescribing instructions at the time (Fiore et al., 2008). Double-blind randomization was conducted using a randomization scheme blocking on gender and race (White vs. non-White). All participants received six counseling sessions (each lasting 10-20 minutes), designed to provide social support and training in problem-solving and coping skills. Bachelor- 
WITHDRAWAL SYMPTOMICS

level case managers provided counselling based on the study protocol and were supervised by a licensed clinical psychologist. The study was approved by the University of Wisconsin Health Sciences Institutional Review Board.

\section{Measures}

Participants completed ecological momentary assessments (EMA) four times a day (once just after waking, once prior to going to bed, and twice at randomly chosen times between waking and sleeping) for 1 week pre-quit and 2 weeks post-quit. We analyzed data from the 2week post-quit period. The EMA prompted participants to rate their withdrawal symptoms within the last 15 minutes using 11 items from the Wisconsin Smoking Withdrawal Scale (Welsch et al., 1999) and 1 item adapted from the Questionnaire of Smoking Urges (Sweeney et al., 1996) to asses 6 symptoms: anxiety (tense or anxious, impatient), craving (bothered by desire to smoke, urge to smoke), sadness (sad or depressed, hopeless or discouraged), irritability (irritable or easily angered, bothered by negative moods such as anger, frustration, and irritability), hunger (hungry, thinking about food a lot), and difficulty concentrating (hard to pay attention, difficult to think clearly). The mean of the 2-items making up each symptom scale was computed.

\section{Data Preparation}

Temporal network models assume stationarity of symptoms such that symptoms do not exhibit a trend in the mean or variance of their intensity across time (Lütkepohl, 2005). We removed linear trends from each symptom by regressing each participants' ratings of symptom intensity on time in the study to remove the linear trend, and then by taking the residuals 
WITHDRAWAL SYMPTOMICS

forward, which is a common practice to handle violations of stationarity in the means. A second assumption of the models used is that there are equal time lags between consecutive measurements (de Haan-Rietdijk et al., 2017). To accommodate this assumption, we did not regress the first measurement of each day on the last measurement of the previous day.

\section{Data Analysis}

We used a vector autoregressive model (VAR) to estimate the dynamic associations among withdrawal symptoms at the group level (Epskamp et al., 2016). Each symptom was regressed on the values of all other symptoms at the previous time point. To accommodate the nested nature of the data, with participants providing multiple reports, a multilevel extension of VAR was used (Bringmann et al., 2013; Lydon-Staley et al., 2019a). The resulting coefficients represent the degree to which changes in one symptom at time $t$ predict changes in another symptom at time $t+1$ for the average individual in the sample.

The VAR approach produces both a temporal and a contemporaneous network. Edges in the temporal network encode information regarding how symptoms at one measurement occasion influence symptoms at the next measurement occasion. This network has the potential to provide information about the directionality of effects (Epskamp et al., 2018), and thus estimates of influence are depicted as directed edges with arrows indicating the direction of effects across time. Edges in the contemporaneous network show associations between symptoms occurring within consecutive measurements and indicate partial correlations between nodes at the same time, controlling for both temporal effects and all other variables within the same measurement occasion. Edges in the contemporaneous network are computed by correlating the residuals of the temporal effects (Dahlhaus \& Eichler, 2003). The 
WITHDRAWAL SYMPTOMICS

contemporaneous network provides insight into the co-occurrence of symptoms, and may also indicate the presence of causal associations occurring on a shorter timescale than the timescale of measurement (Epskamp et al., 2018).

After estimating a group level dynamic withdrawal network, we used the same participants and measures to estimate person-specific dynamic networks of withdrawal. The raw time series data for the 6 withdrawal symptoms were regressed on time in order to remove trends and meet the assumption of stationarity. These data were then within-person standardized so that each symptom for each person had a mean of 0 and a standard deviation of 1 . This standardization was performed to render the coefficients representing different edges in the network comparable to one another (Bulteel et al., 2016). We then modeled each participant's 6symptom time series as a 6-node network using a unified Structural Equation Model (uSEM) (Gates et al., 2010).

The uSEM model is estimated using an iterative search process. A series of models is constructed and improvements to model fit are determined. With each iteration, Lagrange Multiplier tests (Sörbom, 1989) are used to select the edge which, if free to be estimated, would have the maximum improvement on model fit. Model fit is determined according to a predetermined $\alpha$ level on a $\chi$-square distribution with one degree of freedom. Here, as elsewhere (Beltz et al., 2013), we set $\alpha$ to 0.05 . This edge is freed, the model is re-estimated, and a new set of modification indices are calculated. Edges are freed or added until further addition does not significantly improve model fit. Using pompom (Yang, Ram, \& Molenaar, 2018), an R package that implements person-specific uSEM, we estimated person-specific networks specifying how the individual tobacco withdrawal symptoms causally influenced one another from one measurement occasion to the next (lagged) and during concurrent measurement occasions 
WITHDRAWAL SYMPTOMICS

(contemporaneous). A detailed overview of the estimation procedure can be found in the supplement (Study 2: Unified Structural Equation Modeling and Impulse Response Analysis).

To test the self-perpetuating symptom networks hypothesis, we then used impulse response analysis (Lütkepohl, 2005) to provide insight into how the experience of one symptom propagates through the withdrawal symptom network by influencing other, connected symptoms. In this approach, an impulse is given to an individual node and the behavior of the symptom network is observed, through simulation, over many time steps. This dynamical process simulates how a symptom's activity moves through the symptom network along edges when it becomes transiently increased due to an external perturbation. Formally, the impulse response simulation is derived from the uSEM as a one-step-ahead forecasting process through conversion into a vector auto-regression model (Amisano \& Giannini, 2012). The withdrawal network is set in motion by sending an initial impulse to an individual symptom (e.g., craving) and computing how the system evolves over 100 time steps. The time profile, showing how craving intensity changes over the time steps following the initial impulse, is examined. Specifically, the recovery time of craving, defined as the time taken to return to within $+/-0.01$ of equilibrium, is derived via a backward search. We searched backward from the end of the time profile to identify the time step $k$ where the intensity of craving is first outside the $+/-0.01$ boundary. Recovery time is then quantified as the number of time steps from perturbation to equilibrium. Additional detail on impulse response analysis can be found in the supplement (Study 2: Unified Structural Equation Modeling and Impulse Response Analysis).

We next quantified individual differences in the extent to which withdrawal symptom networks facilitate self-perpetuating symptom activity across time by estimating system recovery time. Impulses were given to all 6 symptoms, each time tracking changes in all 6 symptoms. The 
WITHDRAWAL SYMPTOMICS

result is a 6x6 matrix of symptom activity trajectories. We took the mean value of the column means in the $6 \times 6$ matrix as an indication of system recovery time, indicating the average time taken for a symptom to return to equilibrium after perturbation. To explore the extent to which system recovery time may be impacted by treatment, we compared the system recovery time of participants receiving combination therapy during the quit attempt (nicotine patch + nicotine lozenge and buproprion SR + nicotine lozenge conditions) to participants in combination therapy placebo conditions using independent samples $t$-tests. A second independent samples $t$-test was used to determine whether system recovery time differed between participants in the monotherapy conditions (buproprion SR, nicotine lozenge, and nicotine patch) and the associated placebo conditions.

\section{Results}

The edges of the temporal network (Fig. 3A) represent the influence of symptoms at one measurement occasion on symptoms at the next measurement occasion for the prototypical individual in the sample. We find that all estimated edges are positive, such that withdrawal symptoms at one moment increase (rather than decrease) the experience of other withdrawal symptoms (including themselves) at the next moment. Craving has many outgoing edges, indicating that increased craving at one moment has a relatively strong influence on other withdrawal symptoms from moment to moment. Both sadness and irritability have the largest number of ingoing edges, indicating that participants experience increases in sadness and irritability following increases in the experience of other withdrawal symptoms. The majority of symptoms show positive self-loops (edges pointing towards themselves), which indicate that current symptom experiences are predictive of subsequent experiences. Notable is the presence 
WITHDRAWAL SYMPTOMICS

of reciprocal edges between sadness and irritability, and between anxiety and irritability. These reciprocal edges represent positive feedback loops whereby the experience of one symptom (e.g., irritability) increases the experience of another symptom (e.g., anxiety), which in turn reinforces the experience of the original symptom (i.e., irritability). Also notable is hunger, which is disconnected from the other withdrawal symptoms; the lack of incoming or outgoing edges to and from hunger indicates that fluctuations in the experience of hunger over time do not play a large role in reinforcing other withdrawal symptoms.

\section{[FIGURE 3 ABOUT HERE]}

The edges of the contemporaneous network (Fig. 3B) represent the co-occurrence of symptoms, controlling for both temporal effects and the effects of all other variables at the same measurement occasion. Edges are unidirectional (without arrows) but they may indicate causal associations playing out at timescales shorter than the timescale of symptom measurement (Epskamp et al., 2018). Similar to the temporal network, all edges are positive, indicating that when an increase in one symptom is experienced (e.g., sadness) an increase in a second symptom is experienced (e.g., irritability).

We next moved from a group model indicating the temporal associations between symptoms across time for the prototypical individual in the sample to the estimation of personspecific temporal networks. The estimation of person-specific networks requires substantially more data than the estimation of a group model that pools data across individuals. A personspecific model of the associations among the 6 withdrawal symptoms fit the data of 290 individual participants well; the goodness of fit is indicated by at least three of four following 
WITHDRAWAL SYMPTOMICS

criteria: RMSEA $\leq 0.08$, SRMRs $\leq 0.08$, CFIs $\geq 0.95$, NNFI $\geq 0.95$ (Beltz et al., 2013; Yang et al., 2018). Comparing the number of days available for participants for whom a person-specific model fit well relative to those for whom a person-specific model could not be fit well indicate that, in line with previous work (Yang et al., 2018), the length of the time series impacted model fit, with a significantly greater amount of time series data available for participants with good $(M=37.21, n=290)$ versus poor $(M=23.16, n=998)$ model fit $(t(745.72)=21.692, p<0.001)$.

Substantial heterogeneity in symptom network structure is evident (Figure 4A). We quantify individual differences in participants' withdrawal symptom networks and their implications for the extent to which symptom activity becomes self-perpetuating across time due to the interplay among symptoms by using impulse response analysis (Lütkepohl, 2005; Yang et al., 2018). In this approach, we observe how the experience of one symptom spreads across an individual's withdrawal symptom network by simulating an impulse or a perturbation to a specific symptom. When an impulse is given to a symptom, symptom activity increases, and the impulse diffuses through the network, following the directed edges connecting symptoms across time. Eventually, the system returns to a stable state following the perturbation induced by the impulse. The time taken for the system to return to this stable state is referred to as the system recovery time, which can provide an indication for how the network edges maintain withdrawal symptom activity across time (Fig. 4B).

\section{[FIGURE 4 ABOUT HERE]}

The average system recovery time for the sample is $3.18(S D=2.55)$ time steps and exhibits substantial positive skew $(\min =0.89, \max =26.86$, skew $=4.23$ ). We used the common 
WITHDRAWAL SYMPTOMICS

logarithm to reduce the positive skew and remove three outliers (all greater than $3 S D$ s above the mean). Using independent samples $t$-tests we found that participants undergoing combination treatment have faster recovery times relative to participants in a matched placebo condition, $t(26.76)=-2.23, p=0.03$ (Fig. $4 C$ ). There was no significant difference between participants in monotherapy treatment and a matched placebo condition, $t(21.04)=1.00, p=0.33$. Thus, participants in an intensive combination treatment condition showed faster recovery times relative to participants in a matched placebo condition but participants in a less intensive monotherapy condition showed no difference in symptom network recovery time relative to participants in a matched placebo condition.

\section{Discussion}

Smoking cessation is notoriously difficult, with the vast majority of quit attempts ending in relapse (Alterman, Gariti, \& Mulvaney, 2001; Fiore, Bailey, \& Cohen, 2000). Withdrawal symptoms emerge following reductions in smoking that are associated with decreased quit intentions as well as increased smoking relapse (Orleans et al., 1991; Allen et al., 2008; West, Hajek, \& Belcher, 1989). The present study examined the potential utility of a network perspective on tobacco withdrawal, particularly in providing insight into smokers' withdrawal experiences. Using an acute smoking abstinence experimental design, we found that individual symptoms show marked differences in the extent to which their intensity increases in response to acute (16 hours) abstinence. These results complement a body of literature providing evidence for unique time courses associated with individual withdrawal symptom intensity across periods of smoking abstinence (Leventhal et al., 2010; Hendricks et al., 2006; Piasecki et al., 2000; West et al., 1987). These results also indicate that, in contrast to a latent disorder perspective of 
WITHDRAWAL SYMPTOMICS

withdrawal, individual symptoms of withdrawal are not interchangeable. This lack of interchangeability suggests that approaches which treat individual symptoms as important in their own right may be promising.

We accommodated the lack of interchangeability between individual withdrawal symptoms by using a network perspective that has been fruitfully applied to capture the importance of unique symptoms of other disorders, including depression (Fried \& Nesse, 2015), post-traumatic stress disorder (Segal et al., 2019), and, more recently, tobacco withdrawal (Lydon-Staley et al., 2018). In line with recent work introducing the network perspective to tobacco withdrawal (Lydon-Staley et al., 2018), cross-sectional networks indicated many associations among individual symptoms of withdrawal, consistent with the notion of withdrawal as a network of interdependent symptoms. These findings reinforce existing cross-sectional work in tobacco withdrawal but also extend them to a largely African-American sample. Importantly, African Americans are subject to disparities in tobacco-related disease, with African American smokers less likely to initiate a quit attempt and more likely to relapse after quitting relative to smokers identifying as White (Choi et al., 2004).

Further extending previous tobacco network research, we investigated network structure across periods of smoking satiety and abstinence. Tobacco symptom network structure invariance has previously been observed in the context of smoking reduction. Yet, data were collected during treatment with nicotine patch and, as such, treatment may have impacted network stability (Lydon-Staley et al., 2018). The acute smoking abstinence design was a key strength of the present study, providing a stringent test for invariance in symptom structure across states of smoking satiety. No differences in symptom network structure were found across periods of smoking abstinence and satiety. This null effect is encouraging from a treatment 
WITHDRAWAL SYMPTOMICS

planning perspective and, if replicated using temporal networks, presents the possibility of using pre-cessation data to guide interventions during smoking reduction and abstinence.

A key contribution of the present paper was to model the interplay among withdrawal symptoms using experience-sampling data containing temporal information on the moment-tomoment activity of withdrawal symptoms. The edges in cross-sectional networks indicate between-person associations, with strong edges indicating that if an individual experiences high levels of anxiety, for example, they are likely to also experience high levels of anger. This symptom co-occurrence, from a network perspective, is theorized to result from causal associations among symptoms. Yet, between-person associations (i.e., people high in anxiety experience high anger) do not always translate to the within-person associations (i.e., at times when a person is more anxious than usual, they are also angrier than usual; Bos et al., 2017; Hamaker, Dolan, \& Molenaar, 2005). To address this open challenge in the field, we estimated networks in which the edges indicated the within-person association between symptom activity at one time point and symptom activity at the next time point. The temporal networks of withdrawal exhibited substantial interplay among symptoms from moment to moment.

One important insight from the temporal network derives from the sole existence of positive edges, such that if symptom activity was high at one time point, it was more likely to increase, rather than decrease, in activity at the next time point. Indeed, the group temporal network contained numerous potential positive feedback loops that might render symptom activity vulnerable to exhibiting self-perpetuation of symptom activity due to the reverberation of symptom activity through the network. For example, anxiety at one time point was associated with increased irritability and anger at the next time point and, in turn, irritability and anger at one time point was associated with increased anxiety at the next time point. The capacity for 
WITHDRAWAL SYMPTOMICS

one's current emotions to give rise to other emotions has long been emphasized in emotion theory (e.g., Gross \& Muñoz, 1995) and there is empirical support for the moment-to-moment transfer of emotions across time and states (Anand et al., 2016; Lydon-Staley et al., 2019a; Pe \& Kuppens, 2012). Additional findings of interest include the many out-going edges from craving to other withdrawal symptoms, suggesting that the experience of craving at one moment leads to the emergence of aversive states (e.g., anxiety, irritability) at the next moment. Thus, craving may be a useful treatment target, not simply to avoid drug use in response to craving, but also to ameliorate aversive states resulting from its experience (Tiffany \& Wray, 2014). Going forward, it will be important to identify the mechanisms underlying the network edges. In the context of the cross-talk observed between emotions across time, for example, emerging work suggests the relevance of individual differences in the neural correlates of cognitive control as well individual differences in the use of emotion regulation strategies involving the adoption of an accepting and open attitude to one's current experience for understanding the spread of emotions to other emotions across time (Drake et al., 2019; Lydon-Staley et al., 2019a; Lydon-Staley et al., 2019b).

As well as allowing us to construct temporal networks of withdrawal symptoms, the use of experience-sampling data enabled us to probe the notion of self-perpetuating symptom networks and its implications for the experience and treatment of withdrawal by computing person-specific, temporal networks of withdrawal. At the core of network perspectives in psychology are notions of individual differences in the extent to which symptom networks are vulnerable to the reverberation of symptom activity across time as symptoms influence one another. We captured the extent to which withdrawal symptom networks were vulnerable to selfperpetuation capturing the time it took for symptom activity to recover after a simulated increase 
WITHDRAWAL SYMPTOMICS

in activity that spread along the estimated directed paths of the symptom network. Findings showed that combination nicotine replacement smoking cessation treatment was associated with reduced symptom network recovery time relative to a placebo condition. While combination nicotine replacement has been shown to work by reducing craving (Bolt et al., 2012), this research suggests that this treatment may also reduce the time it takes for a smoker to recover and return to baseline levels of withdrawal (i.e., negative affect, cognitive disturbance, craving) after an acute increase in withdrawal symptom activity. This observation encourages future efforts with withdrawal symptom time series of longer length to examine the extent to which treatment encourages smoking cessation by reducing symptom interactions and the perpetuation of aversive withdrawal states across time.

Given evidence for the lack of interchangeability of individual withdrawal symptoms, an important avenue for future work will be to view individual symptoms as important targets for examination and treatment in their own right. Yet, the individual symptoms considered to fall under the purview of withdrawal differ across scales and studies, including those used in the cross-sectional and temporal networks in the present study, which will impact the ability to compare withdrawal networks across studies. However, there is moderate overlap in the symptoms included on the most widely-used withdrawal scales (see Supplemental Study: Content Analysis of Common Withdrawal Scales) suggesting that the field is well-placed to meet this challenge. For context, we note that there is much less consistency in the symptoms falling under the purview of other disorders, with symptom overlap across depression scales, for example, emerging as low in previous work (Fried, 2017). 
WITHDRAWAL SYMPTOMICS

\section{Conclusions}

The present study examined the response of individual withdrawal symptoms to acute smoking abstinence in addition to the cross-sectional, temporal, and person-specific network structure of tobacco withdrawal. In doing so, we find that symptoms of tobacco withdrawal are not interchangeable, that there are substantial, person-specific associations between individual withdrawal symptoms across time, and that smoking cessation treatments may impact the extent to which withdrawal symptom activity lingers across time due to inter-symptom influences.

These findings suggest that a network perspective of tobacco withdrawal is a promising approach to understanding withdrawal and its treatment to help all smokers succeed in quitting smoking. 
WITHDRAWAL SYMPTOMICS

\section{Acknowledgements}

D.M.L. acknowledges support from the National Institute on Drug Abuse (K01-A047417-01A1). D.S.B. and D.M.L. acknowledge support from the John D. and Catherine T. MacArthur Foundation, the Alfred P. Sloan Foundation, the ISI Foundation, the Paul Allen Foundation, the Army Research Laboratory (W911NF-10-2-0022), the Army Research Office (W911NF-14-10679, W911NF-16-1-0474, W911NF-17-2-0181), the Office of Naval Research, the National Institute of Mental Health (2-R01-DC-009209-11, R01-MH112847, R01-MH107235, R21-M MH-106799), the National Institute of Child Health and Human Development (1R01HD08688801), National Institute of Neurological Disorders and Stroke (R01 NS099348), and the National Science Foundation (BCS-1441502, BCS-1430087, NSF PHY-1554488, and BCS-1631550). M.E.P. acknowledges support from the National Institute on Drug Abuse (P50 DA019706), the General Clinical Research Centers Program of the National Center for Research Resources NIH (M01 RR03186), and an Institutional Clinical and Translational Science Award (UW-Madison;

KL2 RR025012). A.M.L. acknowledges support from grant K24DA048160 and RSG-13-163-01 and the American Cancer Society. R.A.S. acknowledges support from grant K24 DA045244. The content is solely the responsibility of the authors and does not necessarily represent the official views of any of the funding agencies. 
WITHDRAWAL SYMPTOMICS

\section{References}

Allen, S. S., Bade, T., Hatsukami, D., \& Center, B. (2008). Craving, withdrawal, and smoking urges on days immediately prior to smoking relapse. Nicotine \& Tobacco Research, 10, $35-45$.

Alterman, A.I., Gariti, P., \& Mulvaney, F. (2001). Short- and long-term smoking cessation for three levels of intensity of behavioral treatment. Psychology of Addictive Behaviors, 15, 261-264.

American Psychiatric Association. (2013). Diagnostic and statistical manual of mental disorders ( $5^{\text {th }}$ ed). Washington, D.C.: American Psychiatric Association.

Amisano, G., \& Giannini, C. (2012). Topics in structural VAR econometrics (2 ${ }^{\text {nd }}$ ed.). New York, NY: Springer-Verlag.

Anand, D., Wilt, J., \& Revelle, W. (2017). Within-subject covariation between depression-and anxiety-related affect. Cognition and Emotion, 31, 1055-1061.

Baker, T. B., Piper, M. E., McCarthy, D. E., Majeskie, M. R., \& Fiore, M. C. (2004). Addiction motivation reformulated: an affective processing model of negative reinforcement. Psychological Review, 111, 33-51.

Bekiroglu, K., Russell, M. A., Lagoa, C. M., Lanza, S. T., \& Piper, M. E. (2017). Evaluating the effect of smoking cessation treatment on a complex dynamical system. Drug and Alcohol Dependence, 180, 215-222.

Bello, M. S., Pang, R. D., Chasson, G. S., Ray, L. A., \& Leventhal, A. M. (2017). Obsessivecompulsive symptoms and negative affect during tobacco withdrawal in a non-clinical sample of African American smokers. Journal of Anxiety Disorders, 48, 78-86. 
WITHDRAWAL SYMPTOMICS

Beltz, A. M., Gates, K. M., Engels, A. S., Molenaar, P. C., Pulido, C., Turrisi, R., ... \& Wilson, S. J. (2013). Changes in alcohol-related brain networks across the first year of college: a prospective pilot study using fMRI effective connectivity mapping. Addictive Behaviors, $38,2052-2059$.

Beltz, A. M., Beekman, C., Molenaar, P. C., \& Buss, K. A. (2013). Mapping temporal dynamics in social interactions with unified structural equation modeling: A description and demonstration revealing time-dependent sex differences in play behavior. Applied Developmental Science, 17, 152-168.

Bolt, D. M., Piper, M. E., Theobald, W. E., \& Baker, T. B. (2012). Why two smoking cessation agents work better than one: Role of craving suppression. Journal of Consulting and Clinical Psychology, 80, 54-65.

Borsboom, D. (2017). A network theory of mental disorders. World Psychiatry, 16, 5-13.

Borsboom, D., \& Cramer, A. O. (2013). Network analysis: an integrative approach to the structure of psychopathology. Annual Review of Clinical Psychology, 9, 91-121.

Bos, F. M., Snippe, E., de Vos, S., Hartmann, J. A., Simons, C. J., van der Krieke, L., ... \& Wichers, M. (2017). Can we jump from cross-sectional to dynamic interpretations of networks implications for the network perspective in psychiatry. Psychotherapy and Psychosomatics, 86, 175-177.

Bringmann, L.F., Elmer, T., Epskamp, S., Krause, R.W., Schoch, D., Wichers, M., Wigman, J.T.W., \& Snippe, E. (2019). What do centrality measures measure in psychological networks? Journal of Abnormal Psychology. Advanced online publication. doi: 10.1037/abn0000446. 
WITHDRAWAL SYMPTOMICS

Bringmann, L. F., Vissers, N., Wichers, M., Geschwind, N., Kuppens, P., Peeters, F., ... \& Tuerlinckx, F. (2013). A network approach to psychopathology: new insights into clinical longitudinal data. PloS one, 8, e60188.

Bulteel, K., Tuerlinckx, F., Brose, A., \& Ceulemans, E. (2016). Using raw VAR regression coefficients to build networks can be misleading. Multivariate Behavioral Research, 51, $330-344$.

Choi, W. S., Okuyemi, K. S., Kaur, H., \& Ahluwalia, J. S. (2004). Comparison of smoking relapse curves among African-American smokers. Addictive Behaviors, 29, 1679-1683.

Cook, J. W., Lanza, S. T., Chu, W., Baker, T. B., \& Piper, M. E. (2017). Anhedonia: its dynamic relations with craving, negative affect, and treatment during a quit smoking attempt. Nicotine \& Tobacco Research, 19, 703-709.

Cramer, A. O., van Borkulo, C. D., Giltay, E. J., van der Maas, H. L., Kendler, K. S., Scheffer, M., \& Borsboom, D. (2016). Major depression as a complex dynamic system. PloS one, 11, e0167490.

Dahlhaus, R., \& Eichler, M. (2003). Causality and graphical models in time series analysis. Oxford Statistical Science Series, 115-137.

de Haan-Rietdijk, S., Voelkle, M. C., Keijsers, L., \& Hamaker, E. L. (2017). Discrete-vs. continuous-time modeling of unequally spaced experience sampling method data. Frontiers in Psychology, 8, 1849.

Epskamp, S., \& Fried, E. I. (2018). A tutorial on regularized partial correlation networks. Psychological Methods, 23, 617-634.

Epskamp, S., van Borkulo, C. D., van der Veen, D. C., Servaas, M. N., Isvoranu, A. M., Riese, H., \& Cramer, A. O. (2018). Personalized network modeling in psychopathology: The 
WITHDRAWAL SYMPTOMICS

importance of contemporaneous and temporal connections. Clinical Psychological

Science, 6, 416-427.

Epskamp, S., Deserno, M. K., \& Bringmann, L. F. (2016). mlVAR: multi-level vector autoregression. $R$ package version $0.3,3$.

Epskamp, S., Cramer, A. O., Waldorp, L. J., Schmittmann, V. D., \& Borsboom, D. (2012). qgraph: Network visualizations of relationships in psychometric data. Journal of Statistical Software, 48, 1-18.

Fiore, M.C., Bailey, W.C., \& Cohen, S.J. (2000). Treating tobacco use and dependence: Clinical practice guideline. Rockville, MD: Department of Health and Human Services.

Fiore, M. C., Jaén, C. R., Baker, T. B., Bailey, W. C., Benowitz, N. L., Curry, S. J., ... \& Henderson, P. N. (2008). Treating tobacco use and dependence: 2008 update. Rockville, MD: US Department of Health and Human Services.

Foulds, J., Russ, C., Yu, C. R., Zou, K. H., Galaznik, A., Franzon, M., ... \& Hughes, J. R. (2013). Effect of varenicline on individual nicotine withdrawal symptoms: a combined analysis of eight randomized, placebo-controlled trials. Nicotine \& Tobacco Research, 15, 18491857.

Fried, E. I. (2017). The 52 symptoms of major depression: Lack of content overlap among seven common depression scales. Journal of Affective Disorders, 208, 191-197.

Fried, E. I., Epskamp, S., Nesse, R. M., Tuerlinckx, F., \& Borsboom, D. (2016). What are'good'depression symptoms? Comparing the centrality of DSM and non-DSM symptoms of depression in a network analysis. Journal of Affective Disorders, 189, 314320. 


\section{WITHDRAWAL SYMPTOMICS}

Fried, E. I., \& Nesse, R. M. (2015). Depression sum-scores don’t add up: why analyzing specific depression symptoms is essential. BMC Medicine, 13, 72 .

Gates, K. M., Molenaar, P. C., Hillary, F. G., Ram, N., \& Rovine, M. J. (2010). Automatic search for fMRI connectivity mapping: an alternative to Granger causality testing using formal equivalences among SEM path modeling, VAR, and unified SEM. NeuroImage, 50, 1118-1125.

Hallquist, M. N., Wright, A. G., \& Molenaar, P. C. (2019). Problems with centrality measures in psychopathology symptom networks: Why network psychometrics cannot escape psychometric theory. Multivariate Behavioral Research. Advanced online publication. doi: $10.1080 / 00273171.2019 .1640103$.

Hamaker, E. L., Dolan, C. V., \& Molenaar, P. C. (2005). Statistical modeling of the individual: Rationale and application of multivariate stationary time series analysis. Multivariate Behavioral Research, 40, 207-233.

Hendricks, P. S., Ditre, J. W., Drobes, D. J., \& Brandon, T. H. (2006). The early time course of smoking withdrawal effects. Psychopharmacology, 187, 385-396.

Holme, P., \& Saramäki, J. (2012). Temporal networks. Physics Reports, 519, 97-125.

Hughes, J. R. (2007). Effects of abstinence from tobacco: Valid symptoms and time course. Nicotine \& Tobacco Research, 9, 3, 315-327.

Hughes, J. R., \& Hatsukami, D. (1986). Signs and symptoms of tobacco withdrawal. Archives of General Psychiatry, 43, 289-294.

Lauritzen, S.L. (1996). Graphical models. Oxford, UK: Clarendon Press. 


\section{WITHDRAWAL SYMPTOMICS}

Leventhal, A. M., Waters, A. J., Moolchan, E. T., Heishman, S. J., \& Pickworth, W. B. (2010). A quantitative analysis of subjective, cognitive, and physiological manifestations of the acute tobacco abstinence syndrome. Addictive Behaviors, 35, 1120-1130.

Lütkephol, H. (2005). New introduction to multiple time-series analysis. Berlin, Germany: Springer.

Lydon-Staley, D. M., Xia, M., Mak, H. W., \& Fosco, G. M. (2019a). Adolescent emotion network dynamics in daily life and implications for depression. Journal of Abnormal Child Psychology, 47, 717-729.

Lydon-Staley, D. M., Kuehner, C., Zamoscik, V., Huffziger, S., Kirsch, P., \& Bassett, D. S. (2019b). Repetitive negative thinking in daily life and functional connectivity among default mode, fronto-parietal, and salience networks. Translational Psychiatry, 9, 1-12.

Lydon-Staley, D. M., Schnoll, R., Hitsman, B., \& Bassett, D. S. (2018). The Network Structure of Tobacco Withdrawal in a Community Sample of Smokers Treated with Nicotine Patch and Behavioral Counseling. Nicotine \& Tobacco Research. Advanced online publication. doi: $10.1093 / \mathrm{ntr} / \mathrm{nty} 250$.

Orleans, C. T., Rimer, B. K., Cristinzio, S., Keintz, M. K., \& Fleisher, L. (1991). A national survey of older smokers: Treatment needs of a growing population. Health Psychology, $10,343-351$.

Pe, M. L., Kircanski, K., Thompson, R. J., Bringmann, L. F., Tuerlinckx, F., Mestdagh, M., ... \& Kuppens, P. (2015). Emotion-network density in major depressive disorder. Clinical Psychological Science, 3, 292-300.

Pe, M. L., \& Kuppens, P. (2012). The dynamic interplay between emotions in daily life: Augmentation, blunting, and the role of appraisal overlap. Emotion, 12, 1320-1328. 


\section{WITHDRAWAL SYMPTOMICS}

Piasecki, T. M, Jorenby, D. E., Smith, S. S., Fiore, M.C., Baker, T. B. (2003). Smoking withdrawal dynamics: I. Abstinence distress in lapsers and abstainers. Journal of Abnormal Psychology, 112, 1, 3-13.

Piasecki, T. M., Niaura, R., Shadel, W. G., Abrams, D., Goldstein, M., Fiore, M. C., \& Baker, T. B. (2000). Smoking withdrawal dynamics in unaided quitters. Journal of Abnormal Psychology, 109, 74-86.

Piasecki, T. M., Fiore, M. C., Baker, T. B. (1998). Profiles in discouragement: Two studies of variability in the time course of smoking withdrawal symptoms. Journal of Abnormal Psychology, 107, 2, 238-251.

Piper, M. E. (2015). Withdrawal: expanding a key addiction construct. Nicotine \& Tobacco Research, 17, 1405-1415.

Piper, M. E., Smith, S. S., Schlam, T. R., Fiore, M. C., Jorenby, D. E., Fraser, D. \& Baker, T. B. (2009). A randomized placebo-controlled clinical trial of five smoking cessation pharmacotherapies. Archives of General Psychiatry, 66, 1253-1262.

Schmittmann, V. D., Cramer, A. O., Waldorp, L. J., Epskamp, S., Kievit, R. A., \& Borsboom, D. (2013). Deconstructing the construct: A network perspective on psychological phenomena. New Ideas in Psychology, 31, 43-53.

Segal, A., Wald, I., Lubin, G., Fruchter, E., Ginat, K., Yehuda, A. B., ... \& Bar-Haim, Y. (2019). Changes in the dynamic network structure of PTSD symptoms pre-to-post combat. Psychological Medicine. Advanced online publication: doi: $10.1017 / \mathrm{S} 0033291719000539$.

Solomon, R. L., \& Corbit, J. D. (1974). An opponent-process theory of motivation: I. Temporal dynamics of affect. Psychological Review, 81, 119-145. 
WITHDRAWAL SYMPTOMICS

Sörbom D. (1989). Model modification. Psychometrika, 54, 371-84.

Sweeney, C. T., Pillitteri, J. L., \& Kozlowski, L. T. (1996). Measuring drug urges by questionnaire: do not balance scales. Addictive Behaviors, 21, 199-204.

Tiffany, S. T., \& Wray, J. M. (2012). The clinical significance of drug craving. Annals of the New York Academy of Sciences, 1248, 1-17.

Toll, B. A., O'Malley, S. S., McKee, S. A., Salovey, P., \& Krishnan-Sarin, S. (2007). Confirmatory factor analysis of the Minnesota nicotine withdrawal scale. Psychology of Addictive Behaviors, 21, 216-225.

van Borkulo, C., Boschloo, L., Borsboom, D., Penninx, B. W., Waldorp, L. J., \& Schoevers, R. A. (2015). Association of symptom network structure with the course of depression. JAMA Psychiatry, 72, 1219-1226.

Welsch, S. K., Smith, S. S., Wetter, D. W., Jorenby, D. E., Fiore, M. C., \& Baker, T. B. (1999). Development and validation of the Wisconsin Smoking Withdrawal Scale. Experimental and Clinical Psychopharmacology, 7, 354-361.

West, R. J., Hajek, P., \& Belcher, M. (1987). Time course of cigarette withdrawal symptoms during four weeks of treatment with nicotine chewing gum. Addictive Behaviors, 12, 199203.

West, R. J., Hajek, P., \& Belcher, M. (1987). Time course of cigarette withdrawal symptoms during four weeks of treatment with nicotine chewing gum. Addictive Behaviors, 12, 199203.

Yang, X., Ram, N., Gest, S. D., Lydon-Staley, D. M., Conroy, D. E., Pincus, A. L., \& Molenaar, P. (2018). Socioemotional Dynamics of Emotion Regulation and Depressive Symptoms: 
WITHDRAWAL SYMPTOMICS

A Person-Specific Network Approach. Complexity, 2018, Article ID 5094179. doi:

$10.1155 / 2018 / 5094179$.

Yang, X., Ram, N., \& Molenaar, P. (2018). pompom: Person-Oriented Method and Perturbation on the Model. R Package version 0.2.0. Available from: https://cran.r-

project.org/package $=$ pompom 
WITHDRAWAL SYMPTOMICS

\section{Figure Captions}

Figure 1. Changes in withdrawal symptom intensity across smoking abstinent and non-abstinent sessions. We characterize the extent to which symptom intensity (y-axis) changes across nonabstinent (blue) and abstinent (red) sessions for each withdrawal symptom (x-axis). Seven of the nine symptoms show increases in symptom intensity and effect sizes range from small to large. Notes: ${ }^{*} p<0.001$.

Figure 2. The network structure of the 9 tobacco withdrawal symptoms during the non-abstinent (A) and abstinent (B) sessions. Blue edges represent positive associations between symptoms. Red edges represent negative associations between symptoms. The network structure is estimated using a Gaussian graphical model. Each edge represents partial correlation coefficients between two variables after conditioning on all other variables. The thickness of the edges reflects the magnitude of the association between two symptoms, with the thickest edge set to a maximum of 0.37 across both networks to facilitate comparisons across measurement occasions. Nodes were placed in a circular layout in order to facilitate visual comparison across sessions.

Figure 3. Dynamic networks of tobacco withdrawal. A. A temporal network in which the nodes (circles) represent symptoms and the edges represent the association between symptoms from one measurement occasion to the next. B. A contemporaneous network in which the edges represent the co-occurrence of symptoms at the same measurement occasion while controlling for temporal effects and the effects of other symptoms. Only green edges are present across both temporal and contemporaneous networks indicating that the experience of symptoms tends to be associated with increased experience of other symptoms. Both temporal and contemporaneous 
WITHDRAWAL SYMPTOMICS

networks are estimated by pooling data across all participants in the sample and, thus, edges represent the prototypical associations among symptoms.

Figure 4. Person-specific networks of tobacco withdrawal. A. Two graphs estimated using a unified Structural Equation Model (41) on experience-sampling data show the temporal associations (edges) among individual withdrawal symptoms (nodes) across time for two different participants. Red edges show negative associations; green edges show positive associations; dashed edges are lagged associations; and continuous edges are contemporaneous associations. Notable is the heterogeneity in network structure across the two participants. B. A sample matrix of recovery times associated with the network on the left in panel $\mathbf{A}$, and estimated using impulse response analysis, indicates the time it takes for a symptom to return to equilibrium after being perturbed. For example, when an increase in anxiety is simulated, it takes 4.92 time steps for anxiety to return to baseline (as indicated by the Anx. -> Anx label). A subsample of three symptoms (anxiety, irritability, and sadness) of the full six symptom matrix is shown to enhance readability. C. Independent samples $t$-tests show higher system recovery times in the symptom networks of participants in the combination therapy placebo condition relative to participants in the active combination therapy condition. No differences in system recovery time emerges for participants in the active or placebo monotherapy groups. Notes: Anx.=anxiety; Irr. $=$ irritablility; Sad. $=$ sadness; ${ }^{*} p=0.03 ; \mathrm{NS}=$ non-significant. 
Figure 1.

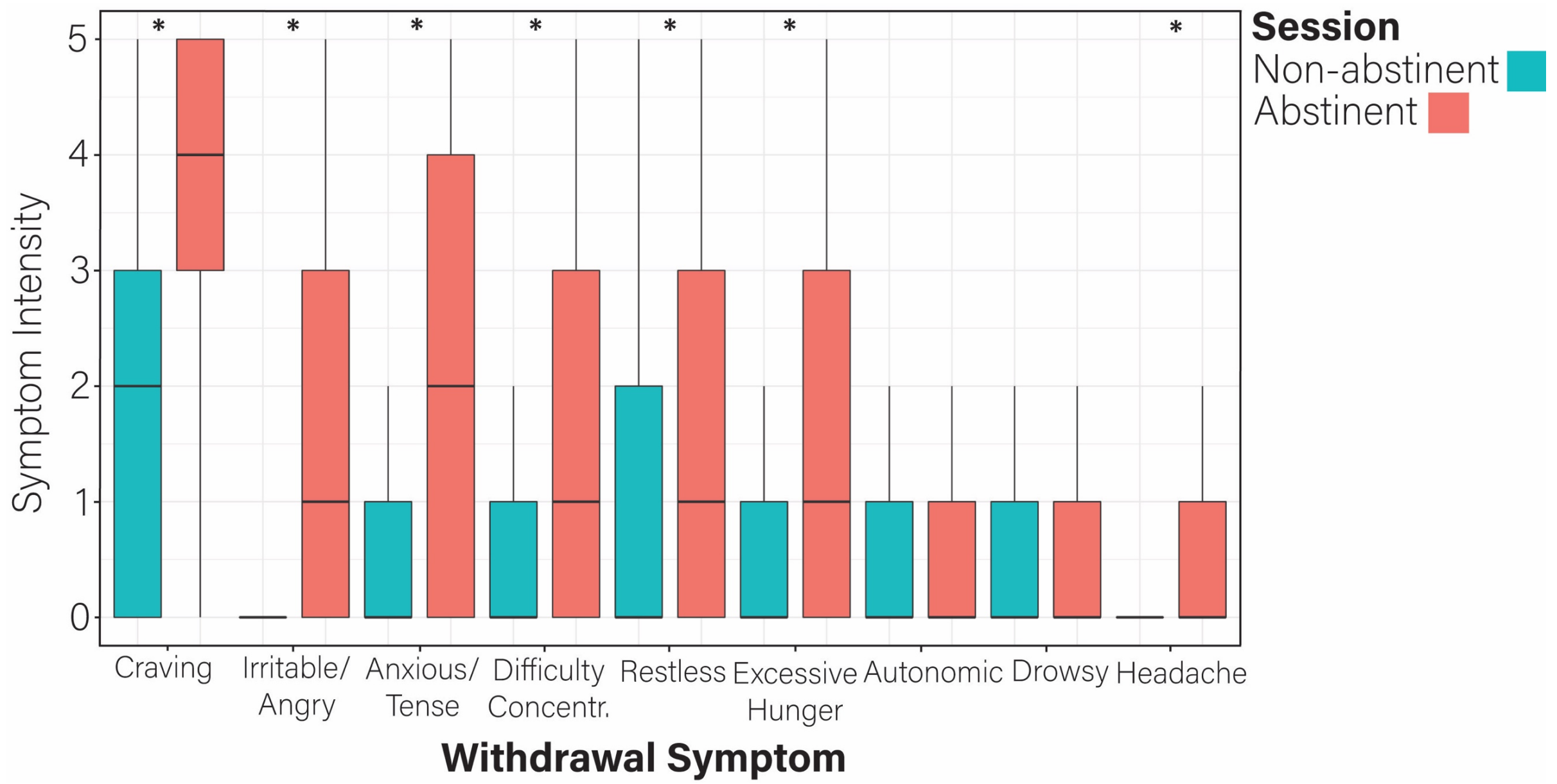




\section{WITHDRAWAL SYMPTOMICS}

Figure 2.

A

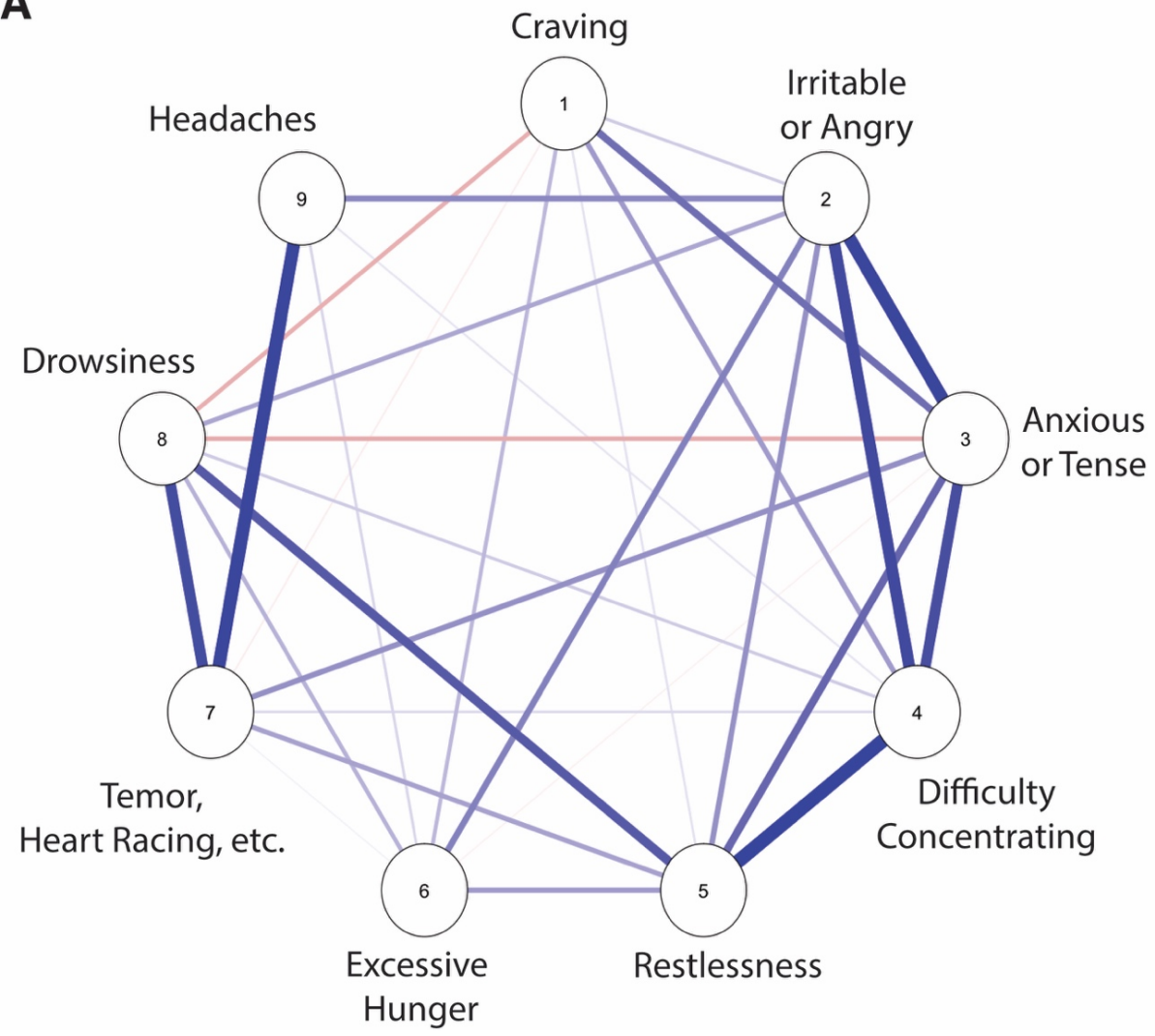

B

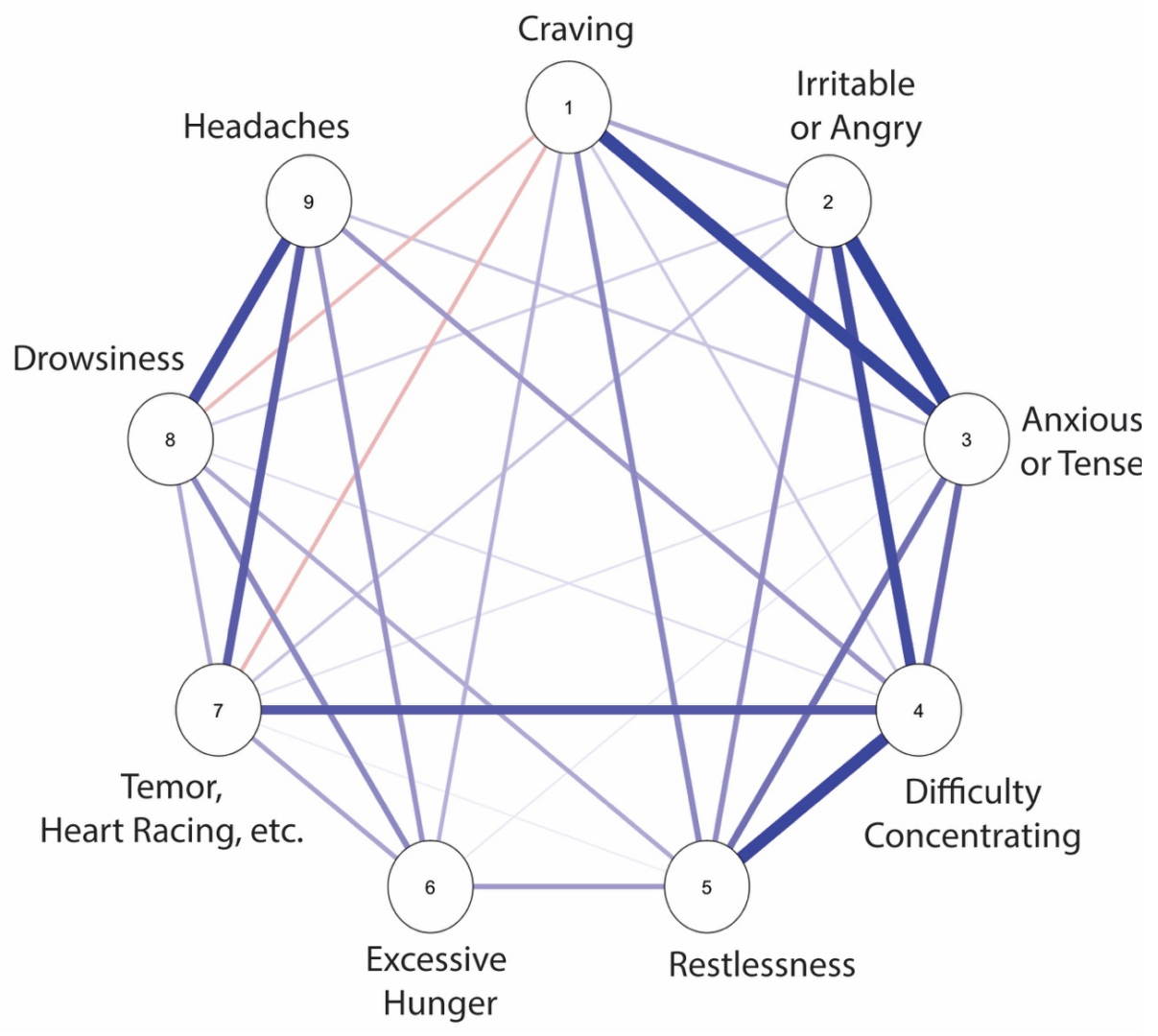


WITHDRAWAL SYMPTOMICS

Figure 3.

A

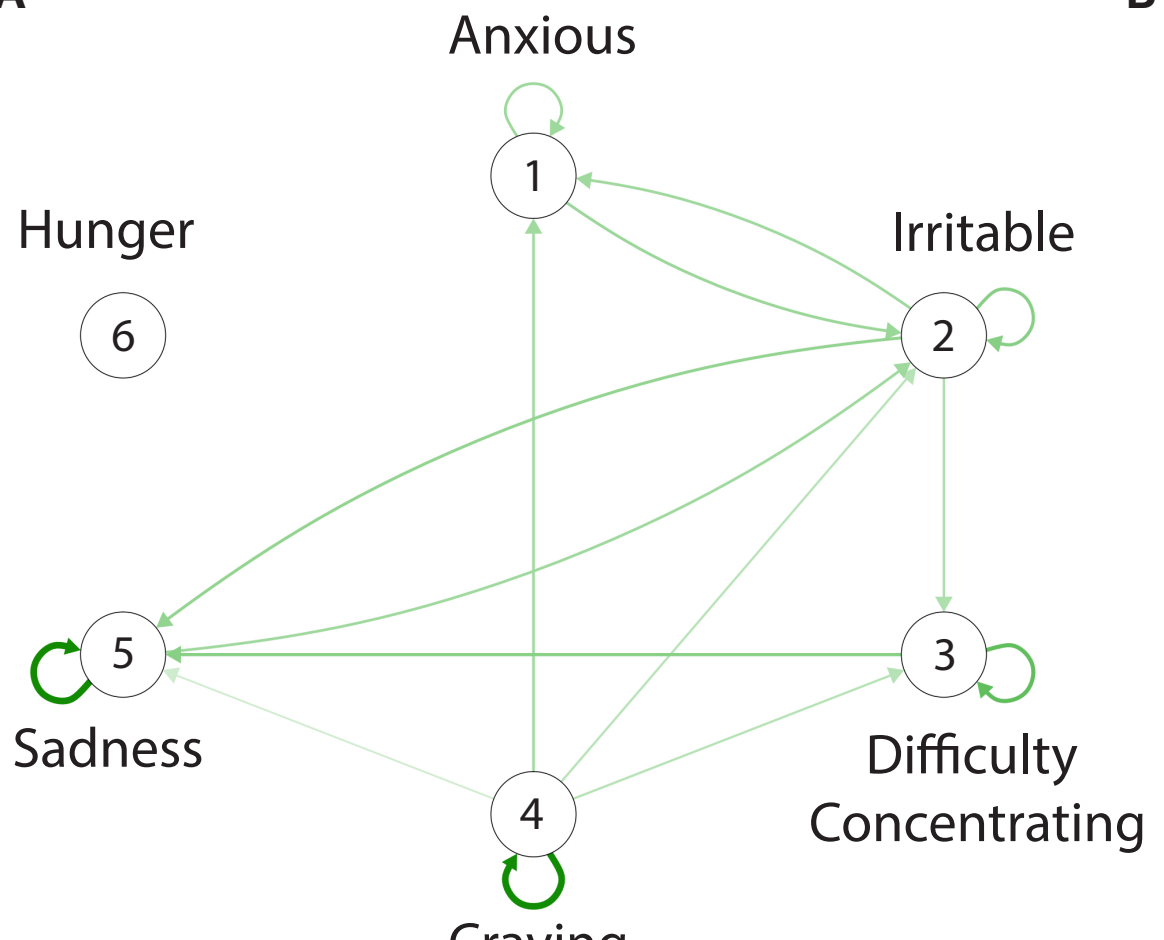

B
Anxious

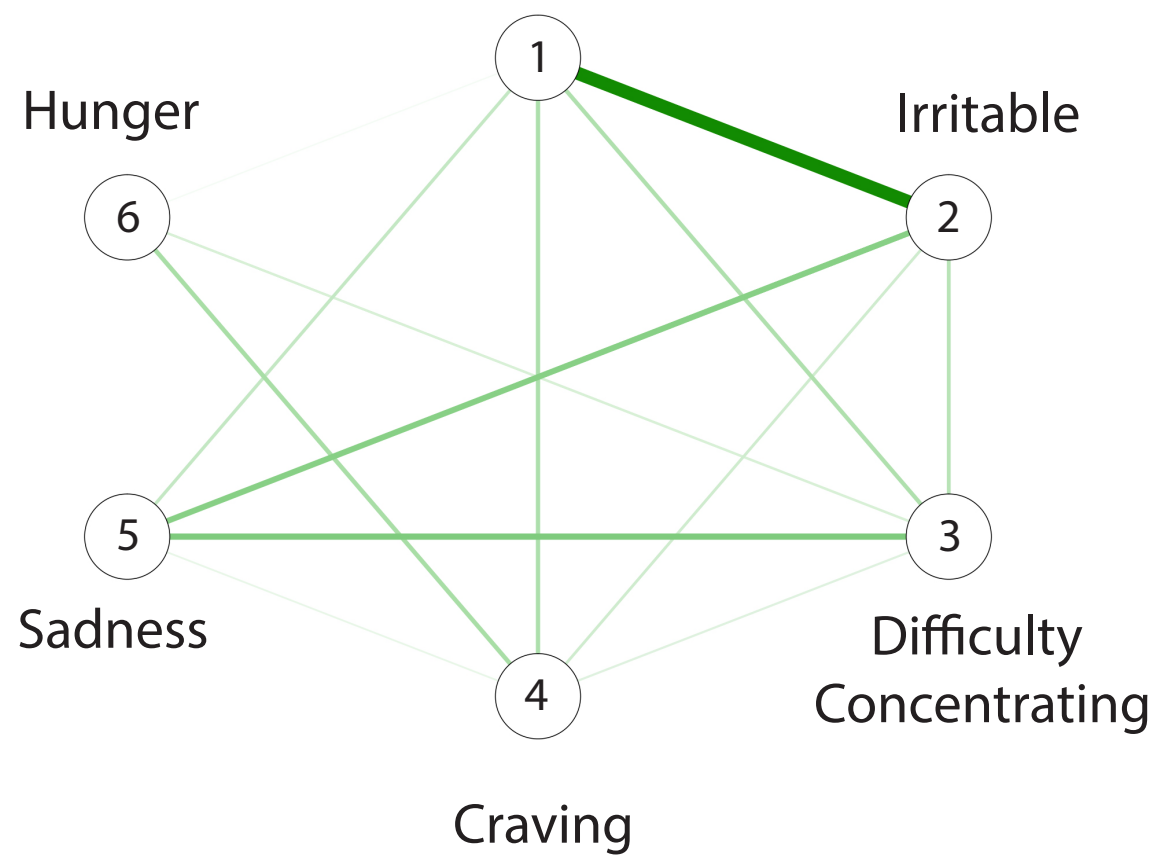


RUNNING HEAD: WITHDRAWAL SYMPTOMICS

Figure 4.

A

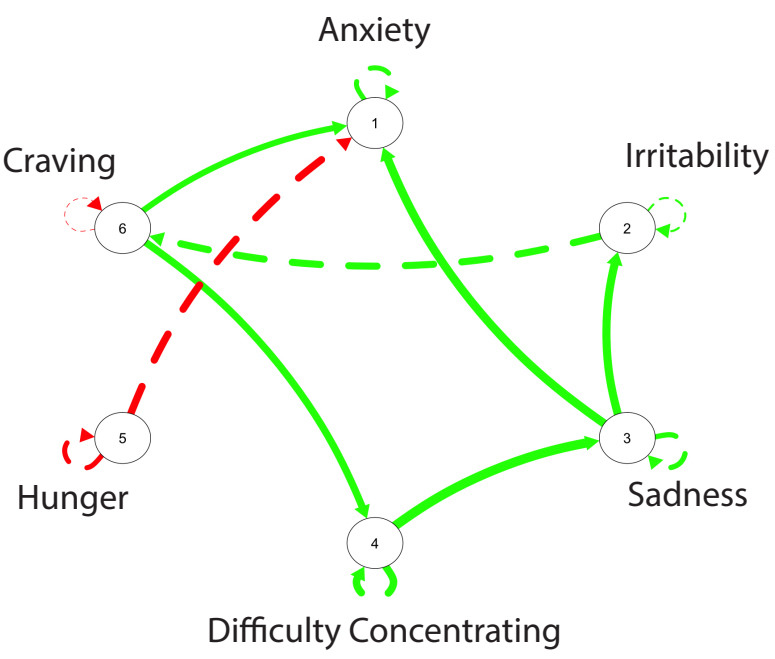

B
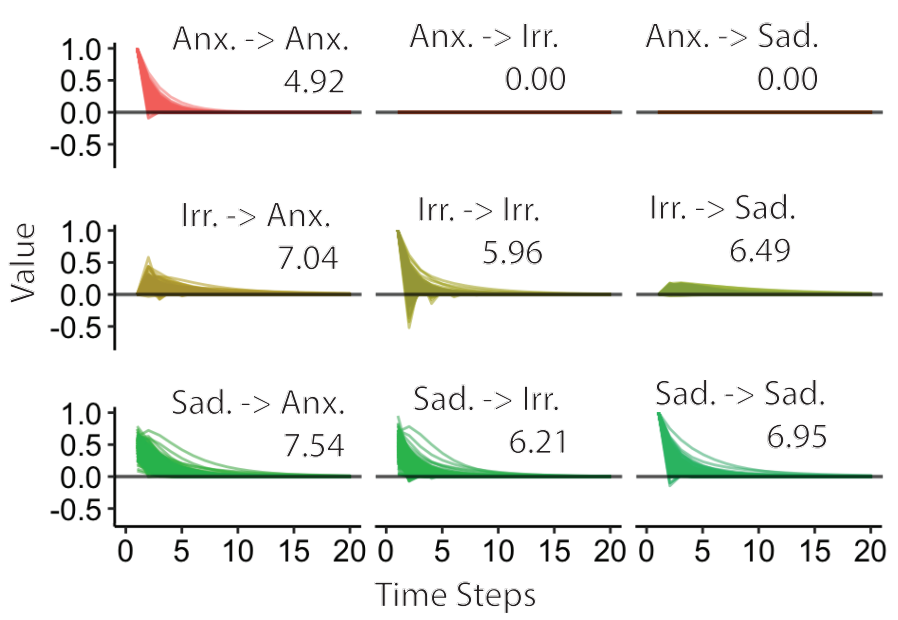

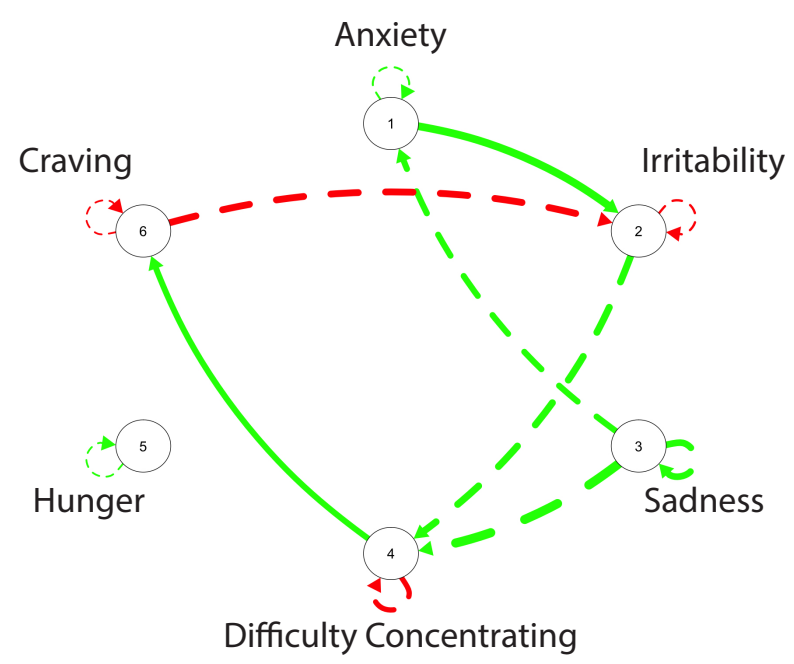

C

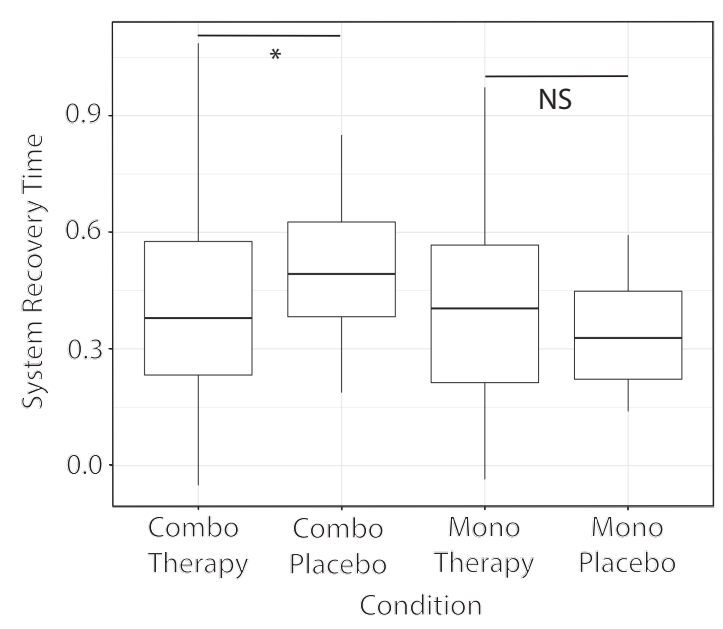

